Gilbert LAZARD

Institut de France, Paris

\title{
MATHESIUS LU À LA LUMIÈRE DE SAUSSURE
}

\section{Introduction}

Vilém Mathesius et Ferdinand de Saussure sont deux figures majeures du renouvellement de la linguistique au début du $X X^{\mathrm{e}}$ siècle. Saussure a professé à Genève ses fameux cours de linguistique générale dans les années 1906-1911, après les avoir longuement médités, et il est mort en 1913 sans les avoir livrés à la publication. C'est à peu près dans le même temps que Mathesius, jeune professeur, a commencé son enseignement et publié ses premiers travaux importants sans connaître encore ceux de Saussure, qui ne lui seront accessibles que bien plus tard, au moment où, à son initiative et sous sa présidence, le Cercle linguistique de Prague commencera son activité. Le titre que j'ai donné au présent exposé ne doit pas donner à penser que j'envisage d'examiner l'ensemble de son œuvre et de la confronter de quelque manière à la pensée saussurienne. Il s'agira seulement de son célèbre article de 1911 (en tchèque; traduction anglaise dans VACHEK, 1964 : 1-32) sur «la potentialité » du langage, celui-là même qui est proposé à la réflexion des participants du présent colloque. Cet article met l'accent sur l'étude synchronique des langues et en même temps sur les variations qu'on constate dans leur fonctionnement. Il me semble que cette double perspective pose des questions qu'il est intéressant d'examiner à la lumière des idées de Saussure.

\section{Mathesius}

L'article de Mathesius est justement renommé comme une des premières manifestations d'une orientation nouvelle en linguistique. Il ouvre des perspectives sur plusieurs points, que je voudrais examiner successivement.

1) C'est un vigoureux plaidoyer pour une linguistique «statique », c'est-à-dire, comme on dira un peu plus tard, synchronique, et plus particulièrement pour l'étude des langues vivantes et de leur fonctionnement, par opposition à la pratique des néo-grammairiens, école dominante à la fin du $\mathrm{XIX}^{\mathrm{e}}$ siècle. Mathesius a luimême affirmé (en 1936) son intérêt spontané et ancien pour cette façon d'aborder l'étude du langage : «Dès le temps de mes études à l'université, j'étais plus attiré par la perspective horizontale [c'est-à-dire synchronique] que par la perspective verticale [c'est-à-dire diachronique] du langage [...] Je n'ai pas hésité à l'affirmer ouvertement plusieurs années avant la publication du Cours de linguistique générale de Saussure. » (cité par VACHEK, 1966 : 137). Il affirme la nécessité de prêter attention à la réalité vivante de l'activité langagière. Les méthodes traditionnelles de la grammaire comparée aboutissent à donner une image très simplifiée de cette réalité. Il appelle à dépasser cette simplification et à observer les phénomènes linguistiques dans toute leur complexité. Il reproche à la linguistique du $\mathrm{XIX}^{\mathrm{e}}$ siècle d'avoir considéré le langage indépendamment des individus parlants et d'y avoir cherché des régularités absolues à l'image des résultats des sciences exactes. Mathesius prend ainsi place parmi ceux qui, depuis que veut se créer une science du langage, se sont efforcés de prendre une conscience exacte de la nature de l'objet de leurs travaux. C'est une longue et difficile tâche, qui peut-être, de nos jours encore, n'est pas complètement achevée. 
2) La complexité des phénomènes langagiers se manifeste dans ce qui dans l'article est appelé leur «potentialité ». Ce mot peut déconcerter. Il désigne la variabilité observable dans ce que nous appellerions aujourd'hui la réalisation des unités linguistiques. Mathesius emploie aussi à plusieurs reprises le mot « oscillation», qui est plus clair. Je préfère pourtant celui de «fluctuation », car « oscillation » évoque un mouvement régulier, périodique, alors que les variations en question sont aléatoires. «Fluctuation » est le terme qu'emploient présentement les linguistes français de l'école de Martinet et il a été usité par Saussure (j'y reviendrai). Je note aussi un passage de l'article où potentiality apparaît comme équivalent de l'allemand Spielraum, littéralement «espace de jeu », c'est-à-dire latitude, éventail de variation, ce qui donne bien une idée du phénomène.

Ces variations, nous dit l'auteur, sont non seulement des différences entre les parlers d'individus différents, mais aussi bien des différences observables dans l'usage d'un même individu. Cette idée est, je crois, largement admise aujourd'hui. BLOOMFIELD a écrit dans son Language (1933: 45) : «Si nous observons assez attentivement, nous verrons que deux personnes - ou plutôt peut-être une même personne à des moments différents — ne parlent jamais exactement de même. » Et je ne sais plus qui a dit que tout énoncé, entendez : toute manifestation concrète de la parole, toute énonciation, est un hapax, c'est-à-dire une occurrence unique. De même que, comme a dit un philosophe antique, on ne se baigne jamais deux fois dans le même fleuve, on ne dit jamais deux fois la même chose.

3) Ces variations aléatoires dans l'exercice du langage se produisent entre certaines limites. Mathesius à plusieurs reprises parle de limites, à propos de la prononciation des phonèmes (en fait il n'emploie pas le terme de phonème dans le passage relatif à la théorie de Kruszewski, mais parle seulement de «l'oscillation de l'articulation des sons » du langage), et aussi à propos d'une étude statistique de la longueur des phrases et du nombre de termes prédicatifs chez certains écrivains anglais. Il ne développe pas cet aspect de la question, mais il importe de le noter, car il deviendra un élément important de la théorie structuraliste et nous y reviendrons.

4) Un autre point important qui reste également implicite, mais qui se laisse entrevoir dans certains des exemples allégués, est le fait que l'examen attentif des variations laisse entrevoir certaines constantes. Ainsi, dans la mesure de la durée des voyelles anglaises, à travers les variations en rapport avec leur environnement, leur position dans le mot et aussi les variations aléatoires des différentes mesures, on voit se dégager des régularités. De même dans l'étude statistique, que je viens de mentionner, sur la syntaxe de certains écrivains anglais, on saisit certaines caractéristiques constantes du style de chacun. De tels résultats, indiqués en passant, illustrent un point d'une grande importance méthodologique, car la méthode qui consiste à chercher l'invariance à travers les variations est d'application très générale en linguistique et sans doute aussi ailleurs.

5) Dernier point, last but not least, Mathesius précise au tout début de son article que les variations qui font l'objet de cette étude ne doivent pas être confondues avec les phénomènes qui constituent l'évolution de la langue: « l'oscillation statique, c'est-à-dire l'instabilité à une période donnée s'oppose à la variabilité dynamique, qui se manifeste dans des altérations survenant au cours du temps ». Traduisons dans des termes plus modernes et plus précis, qui reflètent le progrès de la théorisation structuraliste : les variations aléatoires observées dans 
l'actualisation, en discours, du système synchronique doivent être bien distinguées des altérations du système observables en diachronie. Cette dernière formulation dépasse sans doute la pensée que Mathesius a voulu exprimer en 1911, mais elle représente, je pense, ce dont il avait l'intuition, peut-être encore un peu vague, et qui s'est précisé dans la période suivante, grâce aux travaux du Cercle linguistique de Prague et à l'apport de Saussure.

Cette distinction, ou même, comme dit Mathesius, cette opposition, n'est pas développée dans la suite de l'article. Il ne fait pas état d'un système de la langue qui se trouverait opposé à la manière dont ce système est utilisé dans la communication langagière. Mais il admet implicitement la notion intuitive d'une entité appelée langue, comme la langue anglaise, la langue allemande, la langue tchèque. Il sait bien que les langues changent avec le temps, mais il reconnaît évidemment, sans employer le mot, l'existence «d'états de langue », ce qui signifie qu'on peut distinguer dans l'histoire d'une langue des espaces de temps, des «périodes », comme il dit, telles qu'on peut considérer que dans chacune d'elles la langue ne change pas. C'est en somme la conception traditionnelle. Il critique seulement la représentation trop simple que la linguistique du $\mathrm{XIX}^{\mathrm{e}}$ siècle donne de cette réalité, il souhaite qu'on observe bien plus attentivement les phénomènes qui se manifestent dans l'utilisation de la langue au cours d'une période donnée, et il porte lui-même son intérêt tout particulièrement sur la période actuelle, c'est-àdire sur la langue vivante.

Ce qui est ici en cause, c'est la notion de synchronie. Elle est sous-jacente à tout le développement de Mathesius en 1911, mais elle y est encore peu claire. Saussure en a fait quelques années plus tôt la matière d'une bonne partie de son fameux cours, mais celui-ci n'est encore guère connu hors de Genève. Des échos en sont parvenus jusqu'au Cercle linguistique de Moscou et en particulier à Jakobson avant qu'il ne s'installe à Prague (en 1920). Les idées de Saussure ne seront vraiment connues dans cette dernière ville que vers le milieu des années 1920, particulièrement après l'arrivée de Karcevsky, qui avait fait des études à Genève. Etudiées, commentées, discutées, elles contribueront au mûrissement de celles qui ont fait la réputation du Cercle de Prague.

Je bornerai ici ce bref rappel historique, car mon objet n'est pas de détailler les rapports entre les idées de Saussure et celles du Cercle linguistique de Prague. Je ne me tournerai vers Saussure que pour dire comment je le comprends et pour éclaircir sur cette base les phénomènes sur lesquels Mathesius a voulu attirer l'attention.

\section{Saussure}

La pensée de Saussure peut se résumer en quelques couples de notions, qui représentent autant de distinctions - plutôt que d'oppositions, comme on dit souvent,- - entre langue et parole, synchronie et diachronie, signifiant et signifié, etc., qui forment ensemble une doctrine d'une puissante cohérence. Je crois utile de reprendre brièvement quelques-unes des thèses qui la constituent.

1) Nous avons en français la chance de disposer des deux mots de langue et de langage. Saussure en tire parti pour établir une de ses distinctions fondamentales. Le langage est l'ensemble de tout ce qui est inclus dans l'activité de communication entre les individus au moyen de la parole, c'est-à-dire, non 
seulement les énoncés proférés dans l'acte de communication, avec leur aspect matériel, qui relève de la physique, mais aussi leur soubassement physiologique et psychologique et leur conditionnement social, sans compter toute l'expérience humaine du monde qui s'y exprime. SAUSSURE nous dit (1960:25) :

«Pris dans son tout, le langage est multiforme et hétéroclite, à cheval sur plusieurs domaines, à la fois physique, physiologique et psychique ; il appartient encore au domaine individuel et au domaine social ; il ne se laisse classer dans aucune catégorie des faits humains, parce qu' on ne sait comment dégager son unité. »

Le langage, "pris dans son tout», est donc hétérogène. Cependant, il est évident que l'acte de communication ne peut se produire que si les interlocuteurs se réfèrent à la connaissance qu'ils ont l'un et l'autre d'un même ensemble de signes. Cet ensemble est ce qu'on appelle traditionnellement une langue et que, sous ce même nom de langue, Saussure extrait, c'est-à-dire abstrait, de «l'ensemble hétéroclite du langage »: «Tandis que le langage est hétérogène, la langue ainsi délimitée est de nature homogène : c'est un système de signes, où il n'y a d'essentiel que l'union du sens et de l'image acoustique » (1960: 32).

2) La notion de synchronie est également extraite de l'expérience commune (1960 : 117) :

«La première chose qui frappe quand on étudie les faits de langue, c'est que pour le sujet parlant leur succession dans le temps est inexistante : il est devant un état. Aussi le linguiste qui veut comprendre cet état doit-il faire table rase de tout ce qui l'a produit et ignorer la diachronie. [...] Il ne peut entrer dans la conscience des sujets parlants qu'en supprimant le passé. »

La synchronie ainsi décrite n'existe que dans la conscience des participants de l'acte de parole. Mais Saussure généralise la notion et la promeut au statut d'un concept qui couvre l'ensemble de la communauté linguistique et l'ensemble de la langue. Ainsi conçue, la synchronie est évidemment une abstraction. Dans la réalité concrète d'une langue parlée par une collectivité de locuteurs, il n'y a pas de synchronie véritable. Nous savons bien que les langues changent et qu'elles changent constamment. D'autre part, le système qui sous-tend tous les énoncés est supposé être le même dans la tête de tous les membres de la communauté linguistique : en fait il n'en est jamais ainsi. Il y a quantité de variations selon les lieux, les classes sociales et même le niveau d'éducation et les habitudes de chaque individu.

Saussure le sait bien et il justifie en ces termes l'opération d'abstraction qu'il accomplit (1960: 142) :

«En pratique, un état de langue n'est pas un point, mais un espace de temps plus ou moins long pendant lequel la somme des modifications survenues est minime. [...] Un état absolu se définit par l'absence de changements, et comme malgré tout la langue se transforme, si peu que ce soit, étudier un état de langue revient pratiquement à négliger les changements peu 
importants, de même que les mathématiciens négligent les quantités infinitésimales, dans certaines opérations, telles que le calcul des logarithmes. »

La réduction d'une langue à un système synchronique consiste à négliger délibérément toutes ces variations, les changements incessants dans le temps et les différences dans l'espace entre les individus de la collectivité. Cette opération n'est pas illégitime : elle est pratiquée couramment par les spécialistes des sciences exactes. Elle aboutit en l'occurrence à poser la langue comme un système de même nature, en un sens, que le jeu d'échecs. La comparaison revient à plusieurs reprises dans le Cours de linguistique générale.

3) Toutes les langues sont différentes. Cette constatation est encore d'expérience commune tout comme celles, mentionnées ci-dessus, qui fondent la notion de langue et celle de synchronie. C'est cette observation qui est à l'origine d'un principe saussurien célèbre : l'arbitraire du signe. Les signes qui constituent la langue sont faits de l'union d'une manifestation sensible et d'une représentation à laquelle renvoie cette manifestation: Saussure les nomme respectivement signifiant et signifié. Le signifié est une émission phonique, le signifié un fragment de la représentation du monde. Dire que le signe est arbitraire, c'est dire que l'union d'une certaine émission phonique et d'un certain fragment de la représentation du monde n'est conditionnée par rien dans la substance de cette émission ni dans celle de cette représentation. Si l'association du signifiant et du signifié était déterminée par leurs contenus respectifs, comme tous les hommes ont des corps semblables et que vraisemblablement ils ont grosso modo la même expérience du monde, - au moins dans une certaine mesure et en tout cas au niveau élémentaire (voir aussi plus bas), - toutes les langues seraient pareilles. Le fait qu'elles sont différentes indique qu'il n'y a pas de lien naturel entre une certaine combinaison de sons et de bruits et une certain fragment de la représentation du monde. La liaison entre cet ensemble de phénomènes acoustiques et cet ensemble de phénomènes psychiques, entre signifiant et signifié, est arbitraire. Elle est propre à chaque langue et, inversement, une langue donnée est un ensemble particulier de telles associations. La substance de chacune des deux faces d'un signe, si elle est considérée en elle-même, indépendamment de leur liaison, n'appartient pas à la langue. C'est ce que signifie la formule du Cours de linguistique générale que j'ai citée précédemment: «la langue [...] est un système de signes, où il n'y a d'essentiel que l'union du sens et de l'image acoustique $»$.

4) L'arbitraire se manifeste aussi dans le fait que le contenu substantiel des unités linguistique est variable de langue à langue.

$\mathrm{Au}$ niveau des phonèmes, de ce que Martinet a appelé la «deuxième articulation », la substance est constituée par les capacités phonatoires de l'être humain. Chaque langue opère un choix spécifique parmi ces capacités : autrement dit, elle a son propre système phonologique, qui représente un découpage particulier de cette substance. C'est pourquoi, en phonologie classique, un phonème est défini par ses traits différentiels, c'est-à-dire ceux qui le différencient des autres phonèmes. Les propriétés non différentielles n'ont pas de pertinence, 
elles ne font pas partie du système. Ce qui est proprement linguistique, c'est-à-dire fait partie de la langue, c'est la façon dont la substance phonique est découpée.

Le même principe s'applique aussi au niveau de la "première articulation », c'est-à-dire à celui des signes (grammaire et lexique), mais les relations sont ici incomparablement plus complexes. La substance des signifiés est leur contenu de représentation, Saussure pose cette substance des signifiés comme amorphe, non moins que celle des éléments des signifiants. C'est ce qu'on trouve affirmé catégoriquement dans le Cours de linguistique générale (1960 : 155-156) :

«Abstraction faite de son expression par les mots, notre pensée n'est qu'une masse amorphe et indistincte. [...] Prise en elle-même, la pensée n'est qu'une nébuleuse où rien n'est nécessairement délimité. [...] La substance phonique n'est pas plus fixe ni plus rigide : ce n'est pas un moule dont la pensée doive nécessairement épouser les formes, mais une matière plastique qui se divise à son tour en parties distinctes pour fournir les signifiants dont la pensée a besoin. [...] La langue élabore ses unités en se constituant entre deux masses amorphes. »

Cette affirmation signifie que le champ de la pensée ne comprend pas d'articulations intrinsèques et ne se trouve segmenté que par les frontières arbitraires qui lui sont imposées par la langue, c'est-à-dire par l'association de certaines parties de ce champ avec des signifiants.

Cette thèse extrême peut appeler la discussion et elle a été discutée. Beaucoup de gens répugnent à admettre l'idée que la pensée, prise en elle-même indépendamment de sa structuration par la langue, puisse être à ce point informe. Ils ont peut-être raison. Cependant, la thèse saussurienne ne perd pas pour autant sa légitimité. On trouve en effet dans les Écrits, qui sont de la plume de Saussure lui-même, des notes la présentant sous une forme plus nuancée, qui en fait mieux paraître la signification, notamment celle-ci (2002: 73) :

«Ce qui n'existe pas, ce sont :... les significations, les idées, les catégories grammaticales hors des signes; elles existent peut-être extérieurement au domaine linguistique; c'est une question très douteuse, à examiner en tout cas par d'autres que les linguistes. »

Ce passage - corroboré par d'autres - exprime une position moins catégorique que la formulation du Cours. Je l'entends comme signifiant que nous ne savons pas s'il existe des catégories extralinguistiques et que, en tout cas, il n'appartient pas au linguiste de creuser cette question. C'est en effet justement dans la langue qu'on peut trouver les indications les plus objectives pour déceler les catégorisations de la pensée, si bien qu'il est impossible de les distinguer d'emblée de celles qui pourraient être imputables à la mise en forme linguistique. Dans le doute, le linguiste doit, par précaution méthodologique, procéder comme si la pensée était effectivement une masse amorphe.

C'est un fait d'expérience courante que les unités et catégories grammaticales et lexicales ne se correspondent jamais (sauf rares exceptions) d'une langue à l'autre : les traducteurs le savent bien. Tout se passe donc comme si chaque langue représentait un découpage spécifique de la substance sémantique. Dans ces 
conditions, les unités reçoivent une définition purement différentielle. Un mot (ou un morphème) signifie tout ce que ses voisins ne signifient pas. Le signifié d'une unité est fait de l'ensemble de ses emplois possibles, — ou, comme on dit, de ses «différents sens», — ensemble qui n'est limité que par ceux des unités sémantiquement proches. Ses seules frontières sont celles qui résultent de la présence d'autres signifiés de la même langue.

5) Ainsi, les seules réalités saisissables sont les différences entre les unités du système. Au niveau de la première articulation (celle des unités significatives, des signes), ce sont les limites déterminées, dans le champ de la représentation du monde, pour chaque unité, chaque signe, par l'association d'un signifiant avec une région de ce champ. Cette idée, Saussure l'exprime avec force de différentes manières, par exemple (1960: 166) :

«Dans la langue il n'y a que des différences. [...] La langue ne comporte ni des idées ni des sons qui préexisteraient au système linguistique, mais seulement des différences conceptuelles et des différences phoniques issues de ce système. [...] Un système linguistique est une série de différences de sons combinée avec une série de différences d'idées. »

De même dans les Ecrits (2002 : 29) : «On est obligé de poser comme fait primordial le fait GÉNÉRAL, COMPLEXE et composé de DEUX FAITS NÉGATIFS : de la différence générale des figures vocales jointe à la différence générale des sens qui peuvent s'y attacher. »

L'identité d'une langue réside donc dans la manière dont les deux substances sont découpées par leur association. Comme la substance phonique, c'est-à-dire les capacités phonatoires, est la même chez tous les êtres humains, et comme la substance sémantique, c'est-à-dire l'expérience du monde, est en gros la même ou, en tout cas, doit par principe être regardée comme la même, les différences entre les langues ne sont que celles des configurations imposées aux deux substances. En d'autres termes, une langue n'est pas autre chose que cette double configuration. La langue n'est rien d'autre que l'association indissoluble de deux ensembles de frontières. C'est en ce sens que, selon l'adage bien connu, «la langue est une forme, non une substance ». SAUSSURE le dit clairement (1960 : 157) : «La linguistique travaille donc sur le terrain limitrophe où les éléments des deux ordres se combinent: cette combinaison produit une forme, non une substance. ». Le mot de «frontière » ne figure pas dans le Cours tel qu'il a été publié par Bally et Sechehaye, mais on le trouve dans ce même passage sous la plume d'un des auditeurs : «La linguistique a pour domaine ces phénomènes de frontière » $\left(1974, \mathrm{n}^{\circ} 837\right.$, version $\left.\mathrm{G}\right)$.

La langue ainsi conçue est évidemment une abstraction. Le génie de Saussure a été, à partir d'évidences sensibles à tout le monde et de notions intuitives communes (celle de langue, celle de synchronie, celle de la diversité des langues et du contenu de leurs unités), d'élaborer un concept hautement abstrait. Cette abstraction est assez éloignée de l'expérience concrète, mais elle est conforme aux exigences de l'épistémologie de toute discipline scientifique. L'objet d'une science n'est pas donné. Il doit être construit par une élaboration théorique. Je me réfère ici aux écrits du philosophe épistémologue Gilles-Gaston Granger, qui a passé sa vie à réfléchir aux conditions de la construction des sciences, principalement des 
sciences de la nature, mais aussi occasionnellement aux sciences de l'homme. Il s'exprime ainsi à propos de la définition de l'objet d'une science, quelle qu'elle soit $(1992: 30)$ :

« Le pas décisif de toute démarche scientifique à l'égard d'une expérience, [...] c'est la constitution de concepts qui détermineraient la forme la plus générale des objets qui y entrent, la condamnant à être connaissance rigoureuse, mais approchée.[...] L'exemple le plus trivialement connu, [...,] c'est la réduction galiléo-cartésienne [...] de l'objet «mouvement » à des relations quantitativement formulables entre les concepts de temps, d'espace et de masse. A partir de cette décision ascétique, il est certain que la connaissance des phénomènes ne peut être qu'approchée, en ce sens que l'objet de la mécanique les dépouille de leur richesse intuitive. [...] En revanche, on sait désormais de quoi l'on parle. »

La procédure consiste, par une «décision ascétique », à réduire les phénomènes offerts par l'expérience et à en extraire un objet susceptible d'une «connaissance rigoureuse », quoique «approchée », puisqu'elle néglige délibérément toute une part de la «richesse intuitive » de la réalité concrète. Ce principe vaut pour toute science de la nature; il doit valoir aussi pour les sciences de l'homme.

La langue saussurienne néglige délibérément toutes sortes de variations concrètes. Elle est réduite à un système abstrait (semblable au jeu d'échecs). Elle est en un sens déshumanisée. Mais — ou plutôt : de ce fait même, — elle se prête aux analyses et aux opérations par lesquelles on peut espérer y saisir des régularités structurales, c'est-à-dire des invariants interlangues, des «lois » de la constitution des langues en général, et construire des typologies. Conformément à la formule de Granger, les phénomènes sont «dépouillés de leur richesse intuitive », nous n'en prenons qu'une «connaissance approchée », mais «en revanche on sait désormais de quoi l'on parle ».

Granger, dans sa leçon inaugurale au Collège de France a comparé l'état des disciplines qu'on appelle les sciences humaines à celui de la physique et de la mécanique avant que Galilée et Descartes ne les fondent en tant que sciences au sens propre du terme. Mais il a aussi (antérieurement, 1960: 75) considéré la définition saussurienne de la langue comme comparable à la révolution galiléenne en physique :

«La conception saussurienne de la langue opère donc une double réduction du langage : réduction par rapport à l'histoire (la synchronie passe au premier plan de l'étude linguistique), réduction par rapport au contexte psychosocial (on considère la langue «en elle-même et pour elle-même »). Une telle réduction est aussi radicale que la réduction galiléo-newtonienne du fait physique aux espaces, aux temps et aux masses. »

Je crois que l'abstraction saussurienne est effectivement de nature à permettre le développement d'une véritable science des langues.

Avant de conclure ce bref aperçu de la conception saussurienne de la langue, il faut ajouter une remarque très importante. L'étude de la langue ainsi définie n'est 
qu'une partie de la linguistique, ou bien, si l'on veut réserver à cette étude le nom de linguistique, elle n'est qu'une partie des sciences du langage, comme on dit aujourd'hui. Elle laisse en dehors d'elle l'exercice du langage dans la communication, c'est-à-dire l'actualisation du système de la langue dans l'activité langagière, tout le domaine de ce que Saussure appelle la parole. C'est un domaine différent, Saussure y insiste : «La langue, distincte de la parole, est un objet qu'on peut étudier séparément. » Mais la parole ne saurait être étudiée par la même discipline. L'impossibilité de les aborder ensemble est hautement affirmée (1960 : 38-39) :

« Il serait chimérique de réunir sous un même point de vue la langue et la parole. [...]

Telle est la première bifurcation qu'on rencontre dès qu'on cherche à faire la théorie du langage. Il faut choisir entre deux routes qu'il est impossible de prendre en même temps : elles doivent être suivies séparément.

On peut à la rigueur conserver le nom de linguistique à chacune des deux disciplines et parler d'une linguistique de la parole. Mais il ne faudra pas la confondre avec la linguistique proprement dite, celle dont la langue est l'unique objet. »

En fait, Saussure s'est surtout attaché à l'étude de la langue et le Cours de linguistique générale fait beaucoup moins de place à la parole, mais il ne faut pas oublier cet autre versant de l'étude du langage.

\section{Les fluctuations}

Nous sommes maintenant en mesure de situer plus précisément les phénomènes de fluctuation évoqués par Mathesius. Comme je l'ai rappelé, il prend soin de les distinguer de ceux qui affectent la langue au cours du temps : il ne faut pas confondre, comme il dit, "l'oscillation statique », c'est-à-dire les variations aléatoires, avec la «variabilité dynamique », c'est-à-dire les phénomènes plus massifs qui marquent l'évolution de la langue. Ce sont ces derniers qui ont été étudiés avec prédilection par les néo-grammairiens. En réaction à cette orientation, Mathesius attire l'attention à la fois sur l'intérêt de l'étude des états de langue, c'està-dire de la synchronie, et sur ce que cette synchronie comporte et en même temps dissimule de variation permanente.

La théorisation saussurienne éclaire cette dialectique. Elle confirme et précise la distinction établie par Mathesius. La langue, telle que Saussure la définit par abstraction, étant un système synchronique dans lequel chaque unité s'identifie uniquement par ce qui la fait différente des autres, les traits qu'on peut y apercevoir, mais qui ne contribuent pas à cette différence, ne font pas partie de la langue. Ils ne sont pas pertinents et n'intéressent pas le linguiste en tant que descripteur de la langue, c'est-à-dire du système. Ils font partie de la parole, et c'est là qu'ils prennent toute leur importance. C'est précisément le cas des fluctuations. Elles sont étrangères au système. Elles n'affectent pas les unités de ce système, mais seulement la manière dont celles-ci se réalisent, s'actualisent dans le discours.

Dans un historique de l'école linguistique de Prague, VACHEK (1966:4) a rendu hommage à l'article de Mathesius, «plaidoyer éloquent et convaincant pour 
l'étude synchronique des phénomènes linguistiques, démarche qui ne devait se généraliser dans le contexte de la linguistique mondiale qu'après la publication du Cours de linguistique générale de F. de Saussure.» Il ajoute: «Cependant Mathesius a relevé certains traits du langage qui sont restés cachés à l'ingénieux savant suisse. » Cette dernière remarque concerne, naturellement, les fluctuations. Sur ce point Vachek se trompe. Il serait bien étonnant que ces phénomènes aient échappé à la perspicacité de Saussure. Comme nous avons vu, il avait conscience des phénomènes minimes, mais il les a négligés volontairement, comme l'exigeait le processus d'abstraction auquel il a soumis les phénomènes langagiers. En fait, il mentionne bel et bien les fluctuations, et sous ce terme même, dans les documents publiés récemment (2002: 38; v. aussi 71) : «La latitude qui existe au sein d'une valeur reconnue peut être dénommée «fluctuation». Dans tout état de langue, on rencontre des fluctuations ». Cette affirmation est appuyée d'exemples, notamment celui du $r$ français, qui peut indifféremment se prononcer grasseyé non roulé, grasseyé roulé ou dental, alors que dans d'autres langues ces articulations constituent des entités distinctes. Les fluctuations sont les variations observables dans la réalisation, en discours, d'une unité définie, en langue, par sa valeur différentielle.

J'ai relevé dans l'exposé de l'article de Mathesius le fait qu'il note que les variations dont il fait état se produisent entre certaines limites. Cette notion de limite s'accorde parfaitement avec l'idée saussurienne que la langue n'est qu'un réseau de frontières. Les limites des fluctuations sont exactement les frontières qui constituent le système. Tant que les variations ne les dépassent pas, le système ne change pas : on reste dans la synchronie. Si les frontières qui définissent les unités de langue sont franchies, si les phénomènes de fluctuation débordent le champ qu'elles délimitent, le système n'est plus le même, la langue a évolué. Les variations ne sont plus des faits de parole, mais des faits de langue, qui relèvent de la diachronie, de ce que Mathesius appelle la «variabilité dynamique », qu'il a luimême nettement distinguée des fluctuations.

J'ai dit plus haut les vertus et la nécessité de l'abstraction. La réduction de la langue à un système synchronique est une condition favorable à la découverte de relations constantes, c'est-à-dire en somme de «lois » comparables à celles qu'établissent les spécialistes des sciences exactes. Elle doit permettre de découvrir de telles constantes dans la façon dont sont constituées les langues, autrement dit des « universaux » structurels, c'est-à-dire d'apercevoir, à travers leur diversité pratiquement infinie, ce qu'elles ont véritablement en commun. Elle peut aussi mener à saisir des constantes d'évolution, c'est-à-dire des traits communs dans la manière dont les langues changent, en d'autres termes des universaux diachroniques.

En revanche, cette réduction à l'abstraction laisse échapper, en les négligeant délibérément, une multitude de phénomènes perceptibles dans la réalité concrète. Comme dit l'épistémologue, «A partir de cette décision ascétique, il est certain que la connaissance des phénomènes ne peut être qu'approchée », car on «les dépouille de leur richesse intuitive ». Ces phénomènes négligés n'en méritent pas moins l'examen. En linguistique, ils constituent la vie même du langage dans l'activité de communication. Ils appartiennent au domaine de la parole et doivent être étudiés par les méthodes appropriées à ce domaine. Mais ils peuvent aussi 
parfois s'offrir au linguiste comme un espace de transition entre la parole et la langue. L'étude des fluctuations notamment, dans le jeu des innovations de la parole et le processus par lequel certaines se répandent dans la communauté linguistique et finalement se généralisent et deviennent des nouveautés de la langue, peut, dans certains cas, permettre de saisir concrètement comment se prépare l'évolution du système.

L'observation de la parole selon ses méthodes propres, différentes de celles de la linguistique structurale, est l'autre face de la linguistique, ou, comme dit Saussure, une autre linguistique. C'est aujourd'hui un domaine auquel s'applique avec prédilection un grand nombre de linguistes. Ces deux approches, ou ces deux linguistiques, ont toutes deux leur légitimité au sein du vaste ensemble qu'on appelle les sciences du langage.

\section{BIBLIOGRAPHIE}

BLOOMFIELD, Leonard (1933), Language, New York, Holt, Reinhart and Winston. GrANGER, Gilles Gaston (1960), Pensée formelle et sciences de l'homme, Paris, Aubier.

GRANGER, Gilles Gaston (1987), Leçon inaugurale faite le 7 mars 1987, Paris, Collège de France.

GRANGER, Gilles Gaston (1992), La vérification, Paris, Odile Jacob.

MATHESIUS, Vilém (1911), «O potenciálnosti jevů jazykových », Věstník

Královské české společnosti nauk 1911; trad. angl. in Vachek 1964, 1-32.

SAUSSURE, Ferdinand de (1960), Cours de linguistique générale, Paris, Payot [1916], réimpression.

SAUSSURE, Ferdinand de (1974), Cours de linguistique générale, édition critique par R. Engler, Wiesbaden, Harrassowitz.

SAUSSURE, Ferdinand de (2002), Écrits de linguistique générale, Paris, Gallimard.

VACHEK, Josef (1964), The Prague school reader in linguistics, Bloomington, Indiana University Press.

V ACHEK, Josef (1966), The linguistic school of Prague. An introduction to its theory and practice, Bloomington, Indiana University Press.

\section{ABSTRACT}

Reading Mathesius' 1911 famous article in the light of Ferdinand de Saussure's theorisation highlights the convergence between the two scholars as renovators of the approach to the study of language. It clarifies the notion of synchrony and the dialectical relationship between the system of a language (la langue) and its actualisation in discourse (la parole), which are both underlying in Mathesius' article. 